\title{
Desarrollo y evidencia de validez de una escala multidimensional de riesgo de suicidio y factores protectores en un contexto chileno.
} Development and evidence of validity of a multidimensional scale of suicide risk and protective factors in a Chilean context.

\section{Geraldy Sepúlveda-Páez' \\ (iD) 0000-0002-4693-7937 \\ geraaldyegmail.com}

\section{J. Francisco Santibáñez-Palma ${ }^{2}$}

\section{(iD) 0000-0002-9531-8964}

francisco.palma.santyegmail.com

1 Universidad de Tarapacá, Psicóloga, Arica, Chile.

2 Universidad de Tarapacá, Estudiante de Psicología. Arica, Chile.

${ }^{3}$ Universidad de Tarapacá. Ph. D. Escuela de Psicología y Filosofía, Arica, Chile.
Javier Escudero-Pastén ${ }^{2}$

(iD) 0000-0003-4126-9455

j.escuderopasten @gmail.com

Rodrigo Ferrer Urbina ${ }^{3}$

(iD) 0000-0001-5407-3158

rferrer@academicos.uta.cl

Resumen:

El Suicidio es un grave problema de salud pública, por lo que evaluar su riesgo es fundamental para su prevención. Si bien existen múltiples factores relacionados, se cuentan con pocos instrumentos psicométricos para la medición conjunta de factores protectores y de riesgo, dificultando una comprensión multidimensional del comportamiento suicida. Sobre esta base, el presente estudio tiene como objetivo desarrollar una propuesta de evaluación multidimensional, de rápida aplicación, que permita evaluar tanto factores de riesgo como protectores. Se utilizó un diseño transversal, de tipo instrumental, junto a un muestreo no probabilístico, por disponibilidad, con dos muestras, de adultos jóvenes mayores de 18 años ( $n=405$ y $n=117$ ). El instrumento final se compone de 25 ítems y 5 dimensiones (i.e. ideación suicida, estrategias de afrontamiento enfocadas en la emoción, autoestima, apoyo social percibido y expectativas hacia el futuro). Los resultados indican buen ajuste del modelo, utilizando ESEM, adecuados niveles de fiabilidad ( $\omega>$.8) $(a>0.7)$. Además, se contó con evidencia de validez basada en la relación con otras variables e invarianza métrica entre hombres y mujeres. Los resultados obtenidos, sugieren que las puntuaciones son fiables y presentan evidencias de validez para su interpretación en población equivalente.

Palabras claves: ideación suicida; psicometría; factores protectores; factores de riesgo.

Abstract:

Suicide is a major public health concern, making risk assessment essential for its prevention. Although there are multiple related factors, there are few psychometric tools for the joint measurement of both protective and risk factors, making a multidimensional understanding of suicidal behaviour difficult. On this basis, the present study aims to develop a quickly applicable, multidimensional assessment approach to evaluate both risk and protective factors. A cross-sectional, instrumental design was used, together with a nonprobabilistic, availability sampling, with two samples of young adults over 18 years of age ( $n=405$ and $n=117)$. The final measure is composed by 25 items and 5 dimensions (i.e. suicidal ideation, emotion-focused coping strategies, self-esteem, perceived social support and expectations towards the future). Results show good model fit, using ESEM, adequate levels of reliability $(\omega>.8)(a>0.7)$. In addition, there was evidence of validity based on the relationship with other variables and metric invariance between men and women. Results suggest that the scores are reliable and present evidence of validity for their interpretation in equivalent populations.

Keywords: suicidal ideation; psychometrics; protective factors; risk factors. 


\section{Introducción}

El suicidio es un problema de salud pública que afecta a un segmento importante de la población mundial, observándose aproximadamente, 800.000 muertes anuales por dicha causa (Organización Mundial de la Salud [OMS], 2019). En los países que componen la Organización para la Cooperación y el Desarrollo (OCDE), el suicidio es una de las mayores causas de muerte prematura, produciendo más de 152.000 muertes durante el año 2016 (Organización para la Cooperación y el Desarrollo Económico; [OCDE], 2019). A nivel mundial las tasas de suicidio han tenido un aumento del $60 \%$ en los últimos 45 años, lo que supone una tasa de mortalidad de 16 personas por cada 100.000 habitantes (Echávarri et al., 2015b). Si bien la mayoría de las investigaciones sobre suicidio se realizan en Europa y Norte América, la prevalencia de intentos de suicidio en Latinoamérica y el Caribe ha crecido sustancialmente en la última década (Cai et al., 2020), alcanzando, en 2015, un total de 45.800 muertes anuales, siendo más prevalente en hombres ( 8.39 cada 100000 habitantes) que en mujeres (2.12 cada 100000 habitantes) (Mascayano et al., 2015).

Dentro de los países que componen la $\mathrm{OCDE}$, Chile es el segundo país con los mayores incrementos en las tasas de suicidio, teniendo un crecimiento de un $90 \%$ entre los años 1990 y 2011 (OCDE, 2014) y presentando, a 2017, una tasa de 10.7 casos por cada 100.000 habitantes. Si bien, las tasas de suicidio resultan, por sí mismas, alarmantes, el impacto de esta problemática es mayor si se considera que los intentos de suicidios son aún más prevalentes, y que, quienes tienen al menos un intento de suicidio, poseen un riesgo de muerte 100 veces superior a la población general (Echávarri et al., 2015b). La tercera Encuesta Nacional de Salud (2017), mostró que la ideación suicida se presenta en el $2.2 \%$ de la población y los intentos de suicidio alcanzan el $0.7 \%$ de la población nacional.

Entre los grupos de riesgo, el rango etario de 15 a 29 años es uno de los principales afectados, siendo el suicidio, la segunda causa de muerte (Mokdad et al., 2016; OMS, 2019). Este rango etario se divide en dos grupos que tienen características evolutivitas que los hacen más propensos a los intentos de suicidio: 1) adolescencia media y tardía, caracterizada por cambios biológicos, psicológicos y sociales, los cuales suelen ser repentinos y bruscos, generando inestabilidad en el adolescente (Ortiz et al., 2019; Sanchez-Teruel y Robles-Bello, 2014); y 2) adultez emergente, etapa en el cual se presentan nuevos desafíos, como el logro de mayor autonomía, asumir responsabilidades académicas y responder a expectativas personales y sociales. Exigencias que se encuentran asociados a problemas de salud mental (Arnett, 2018; Rossi et al., 2019). 
Existen múltiples factores que incrementan la probabilidad de cometer un intento de suicidio (e.g. económicos, biológicos, psicológicos, sociales), es decir factores de riesgo (Akhtar et al., 2020; Bachmann, 2018). Dentro de estos factores de riesgo, destacan el abuso de sustancias, depresión, ansiedad, enfermedad o discapacidad crónica, aislamiento social y desesperanza (Siu, 2019; Turecki y Brent, 2016; Turecki et al., 2019). De igual manera, algunas estrategias de afrontamiento (e.g. evitación, enfocadas en la emoción) han sido relacionadas generalmente con tasas más altas de autolesiones y sucidio (Guerreiro et al., 2013). Si bien, todas estas características muestran una relación con el suicidio, el factor que ha evidenciado un mayor impacto sobre el suicidio es la ideación suicida (Turecki y Brent, 2016; Turecki et al., 2019; Zhang et al., 2017).

La ideación suicida (en adelante IS), refiere a la aparición de pensamientos y sentimientos vinculados con planificar o terminar con la propia existencia (Crosby et al., 2011; DenisRodríguez et al., 2017; O'Connor y Nock, 2014; Turecki et al., 2019). Algunos autores (e.g. Cañón y Carmona, 2018; King y Kowalchuk, 1994) extienden esta definición, señalando que la IS no se limitan a pensamientos sobre quitarse la vida, sino que implican procesos cognitivos y estados afectivos sobre falta de sentido vital, preocupaciones sistemáticas y/o delirantes, referidas a la autodestrucción, muerte y elaboración de planes concretos. Según el metaanálisis realizado por Liu et al. (2020), la IS varía en severidad desde la ideación pasiva, referida al deseo de estar muerto, a la ideación activa, referida al deseo de suicidarse.

Las estrategias de afrontamiento (en adelante EA) refieren a un conjunto de recursos y esfuerzos tanto cognitivos como comportamentales, orientados a resolver problemas, reducir o eliminar la respuesta emocional o modificar la evaluación inicial de la situación (FernándezAbascal y Palmero, 1999; Lazarus y Folkman, 1986). En la literatura se ha señalado que las EA adaptativas (e.g. autocuidado, socialización, resolución de problemas) funcionan como protectoras de la ideación suicida y eventuales crisis (Simon et al., 2016). En contraste, se ha evidenciado que un inadecuado funcionamiento o algunos tipos de EA presentes en jóvenes y adultos se asocian con un mayor riesgo suicida (Interian et al., 2019; Stanley et al., 2017). En este sentido, las EA enfocadas en la emoción (en adelante EAE), son estrategias centradas en minimizar los resultado emocionales del problema al que la persona se enfrenta (Lazarus y Folckman, 1986), estas han sido relacionadas con un mayor riesgo suicida, representando así un factor de riesgo a considerar, el cual se vuelve importante para los fines de este escrito (Ambrus et al., 2020; Solano et al., 2019).

Aunque la identificación de factores de riesgo ha sido un aspecto clave para la elaboración de estrategias preventivas de la conducta suicida, este enfoque tradicional no ha producido el impacto esperado (Chan, 2016; Steeg, 2018; Surgenor, 2015), lo cual ha impulsado en estos 
Desarrollo y evidencia de validez de una escala multidimensional de riesgo de suicidio y factores protectores en un contexto chileno.

últimos años la elaboración de acciones focalizadas en fomentar el desarrollo de factores protectores, los que aportarían a una mayor comprensión del fenómeno, así como un mejor uso de las estrategias preventivas (Deuter et al., 2020; Sanchez-teruel y Robles-Bello, 2014; Surgenor, 2015).

Los factores protectores refieren a características personales y ambientales que disminuyen los efectos negativos de aquellas circunstancias inmanejables para una persona, aumentando la capacidad de resiliencia, mejorando el sistema de relaciones a nivel interpersonal e intergrupal (Andrade, 2012; Velásquez et al., 2002). Dentro de los factores protectores se han destacado, principalmente, variables psicológicas como autoestima elevada, capacidad de resolución de problemas, expectativas de futuro, inteligencia emocional, satisfacción con la vida, percepción de apoyo social, etc. (Deuter et al., 2020; Echávarri et al., 2015; Ronean et al., 2014). Sin embargo, dentro de todos los factores estudiados, para el propósito del presente instrumento, toman un interés central las características psicológicas que refieren a aspectos susceptibles de intervención, específicamente: autoestima, apoyo social percibido, estrategias de afrontamiento y expectativas hacia el futuro?

La autoestima (en adelante, $\mathrm{AU}$ ) refiere a una evaluación emocional positiva o negativa que realiza el individuo sobre sí mismo y sus capacidades (Rosenberg, 1965; Smith et al., 2014). La baja autoestima se ha identificado como un factor altamente relacionado con el riesgo suicida (Soto-Sanz et al., 2019; O'Connor et al., 2014), mientras que la autoestima elevada, se ha relacionado con una mayor calidad de vida, así como desarrollo de comportamientos adaptativos y satisfactorios con la vida (Manani y Sharma, 2013; Brausch y Decker, 2014; Cáceda, 2014).

El apoyo social percibido (en adelante, ASP) refiere a la evaluación cognitiva de estar conectados con otros y de recibir soporte en situaciones de necesidad (Barrera, 1986; Usta, 2012). Es considerado un factor determinante en la comprensión de la conducta ante eventos estresantes (Lu y Hampton, 2016). Se ha identificado el ASP como un factor de riesgo de ideación suicida y conducta suicida, cuando la percepción es de carácter negativo (BonillaCruz et al., 2018; Durkheim, 1870; Otzen, et al., 2020). A su vez, el ASP se ha relacionado con aspectos protectores, cuando la percepción de disponibilidad de apoyo

1 Es necesario señalar que estos conceptos poseen múltiples definiciones en la literatura, muchas de las cuales son multidimensionales, observándose, por ejemplo, definiciones de autoestima de hasta 4 dimensiones (e.g.Fleming y Courtney, 1984; Schütz et al., 2006; Shavelson et al., 1976) y definiciones de estrategias de afrontamientos con 6, 9 y hasta 14 dimensiones, (e.g. Calver, 1997; Galiana et al., 2020; Lazarus y Folkman, 1984, 1988; Rosenstiel y Keefe, 1983 ). Sin embargo, dado que el propósito de esta propuesta es desarrollar una escala breve, para estudios correlacionales o acciones de tamizaje, y que todos estos conceptos también poseen definiciones unidimensionales (e.g. Austenfeld y Stanton, 2004; Botella et al, 2018; Calderón-de la Cruz et al., 2019; 1965; Eryılmaz, 2011; Pedrosa, Garcia-Cueto et al., 2012; Roseenberg, 1965), se optó por utilizar las definiciones más simples (i.e. unidimensionales). 
social mitiga el impacto adverso de situaciones estresantes, disminuyendo la probabilidad de sufrir problemas de salud relacionados con el estrés o conductas suicidad (Cohen y Wills, 1985; Demirtepe-Saygili y Bozo, 2011; Evans et al., 2013; Heberle et al., 2015; Otzen et al., 2020; Raffaelli et al., 2013).

Las expectativas hacia el futuro (en adelante EHF) refieren al grado en que la persona espera que ocurra un evento, orientando la conducta y su desarrollo a través de la influencia en la planificación y establecimiento de objetivos futuros (Bandura, 2001; Seginer, 2009; Yu et al., 2018). De esta manera las EHF relacionadas con la esperanza (e.i, una cognición positiva orientada al futuro) han sido consideradas de relevancia para la prevención del suicidio, ya que presentan una relación inversa respecto al riesgo suicida (Chang et al., 2017; Cheavens et al., 2016; Hirsch y Kelliher Rabon, 2015; Huffman et al., 2016 como se cita en Yu et al., 2018).

La evaluación de los comportamientos vinculados con el suicido no es una tarea sencilla, sin embargo, para brindar una adecuada estrategia de prevención es necesario poder identificar y estudiar apropiadamente los factores influyentes en los comportamientos vinculados con el suicidio. En Chile el programa nacional de prevención del suicidio (MINSAL, 2013) contempla cinco instrumentos: Escala de desesperanza de Beck (Beck et al., 1974); Escala de ideación suicida (SSI) (Beck, Steer y Ranieri, 1988); Escala de intención suicida de Pierce (Pierce, 1977); Escala de suicidalidad de Okasha (Okasha et al., 1981); y Escala sad persons (Patterson et al., 1983), todos ellos se encuentran desactualizados y se limitan a identificar factores de riesgo, restando otras perspectivas relevante en la comprensión del fenómeno. En este escenario, parece relevante ampliar a una valoración multifactorial del fenómeno, lo que implica disponer de instrumentos multidimensionales, de alcance masivo, accesibles, de bajo costo y que cumplan los requisitos éticos mínimos para sustentar sus conclusiones y decisiones, derivados del proceso de medición (AERA, APA y NCME, 1999, 2014), de modo de reforzar el desarrollo de evaluaciones o diagnósticos.

Si bien, existen múltiples instrumentos de medida que evalúan la ideación suicida (e.g Alarcón-Vasquez et al., 2019; Beck et al., 1988; Fonseca-Pedrero y Albeniz, 2020; Okasha et al., 1981; Paykel et al., 1974), incluyendo algunas propuestas que incorporan factores protectores (Echávarri et al., 2018; Linehan et al., 1983; Ponce-Garcia et al., 2015; Stanley et al., 2017), los instrumentos localizados, que incluyen factores protectores, presentan algunas limitaciones para el contexto nacional: (1) la mayoría de los cuestionarios han sido desarrollados en idiomas extranjeros, lo cual puede presentar sesgos culturales que no son subsanables desde los procesos de traducción (Krach et al., 2016); y (2) ninguno de ellos cuenta con evidencias de validez para su uso en población chilena. Por tanto, el presente estudio 
tiene por propósito desarrollar una propuesta de evaluación que contemple tanto los factores de riesgo (e.i., a ideación suicida, estrategias de afrontamiento enfocadas en la emoción), como los factores protectores (e.i., autoestima, apoyo social y expectativas hacia el futuro) en jóvenes y adultos jóvenes en un contexto chileno.

\section{Método}

\section{Diseño y participantes}

La presente investigación corresponde a un estudio por encuestas, transversal, de tipo instrumental.

El muestreo utilizado fue no probabilístico, por redes sociales y bola de nieve. La muestra quedo constituida por 405 jóvenes y adultos jóvenes, donde el $51.4 \%$ ( $n=208$ ) de los encuestados declaran residir en la ciudad de Arica, un $12.6 \%(n=51)$ en Concepción y un $9.6 \%$ ( $n=39)$ en la región metropolitana de Santiago. El $51.4 \%(n=208)$ se identificó como mujer, el $45,2 \%$ ( $n=183$ ) como hombre y el $1,5 \%(n=6)$ con otra categoría de género. La edad media de los participantes fue de $23(\mathrm{DT}=6,39)$. Respecto al consumo de sustancias en el último año, el $93.4 \%$ ( $n=323)$ de los encuestados reporta haber consumido alcohol, un $51.2 \%$ ( $n=177)$ tabaco y un $49.7 \%(n=172)$ marihuana.

Adicionalmente, con la finalidad de incorporar evidencias de validez basada en la relación con otras variables, se obtuvo una segunda muestra adicional, a partir de un muestreo no probabilístico, por redes sociales y bola de nieve, de 117 jóvenes y adultos jóvenes. La muestra presento las siguientes características, el $65.0 \%$ ( $n=76)$ se identificó como mujer, el 32,5\% ( $n=38)$ como hombre y el $2,6 \%(n=3)$ con otra categoría de género. La edad media de los participantes fue de $23(\mathrm{DT}=6,0)$.

\section{Instrumento}

Escala Multidimensional de Riesgo Suicida (en adelante EMRS): Es una escala desarrollada para evaluar la ideación suicida y factores protectores relacionados con orientación suicida. El cuestionario, en su versión final, está compuesto por 25 ítems, que evalúan 5 dimensiones: 1) Ideación suicida (ID; ítems = 7); 2) Estrategias de afrontamiento emocionales (EAE = 5 ítems); 3) Autoestima ( $\mathrm{Au}=5$ ítems); 4) Apoyo social percibido (ASP= 3ítems); Expectativas hacia el futuro (EHF = 5 ítems). Los reactivos están presentados en formato Likert de 4 categorías ordenadas ( 1 = "Totalmente en desacuerdo" - 4 = "Totalmente de acuerdo"). 
En primera instancia se generaron 15 reactivos por cada dimensión evaluada (i.e. 75 reactivos en total), los cuales se sometieron a una depuración de redacción y contenido mediante jueces expertos (2 psicólogos clínicos y un doctorando con experiencia en psicometría), teniendo dos criterios de valoración para cada ítem: 1) La pertinencia de los reactivos respecto su respectiva operacionalización; 2) La claridad de estos, en términos de coherencia y redacción, como el objetivo del estudio era generar una escala breve, para la retención de los ítems el criterio de exigencia fue elevado, utilizándose una valoración $(-1,0,1)$ y solo se conservaron aquellos ítem que obtuvieron promedios mayores a 0 . En base a las revisiones, se generó una versión de 34 reactivos. Posteriormente se realizó la aplicación mediante la plataforma online Google Forms, a partir de esta aplicación se realizó una depuración basada en la consistencia interna y el análisis de los modelos factoriales. Finalmente, la escala quedó conformada por 25 ítems.

Escala de Satisfacción con la Vida (SWLS) Diener et al. (1985) crearon una escala multi-item, que mide la satisfacción con la vida como un proceso cognitivo de juicio (Muñoz de Aranillas et al., 2010; Martínez et al., 2004). Para este estudio se utilizó la versión SWLS-C, la cual, presenta los ítems traducidos al español y de manera simplificada, para su aplicación en población general, en niños y menores de edad (Álvarez et al., 2018). Contiene 5 ítems, con respuestas tipo Likert entre 1 (muy en desacuerdo) a 5 (muy de acuerdo), con una puntuación de 5 a 25 puntos, a mayor puntaje mayor Satisfacción con la vida. La escala en su aplicación en población chilena presenta un Alpha de Cronbach de 0.815 y una correlación de ítem-total entre 0.51 y 0.7 (Álvarez et al. 2018).

\section{Procedimiento}

La aplicación del cuestionario se realizó mediante la plataforma online Google Forms. Al inicio del cuestionario se proporcionó un consentimiento informado, donde se establecían los objetivos de la investigación, la confidencialidad, anonimato y los derechos de los encuestados, la participación fue totalmente voluntaria con el requisito de tener 18 años o más. El cuestionario incluyó preguntas sociodemográficas (e.g. edad, identidad de género, lugar de residencia), los participantes fueron contactados a través de redes sociales masivas (i.e. Facebook, Twitter e Instagram). Finalmente, siguiendo el mismo procedimiento, se realizó una segunda aplicación a una nueva muestra, utilizando la versión final del instrumento e incluyendo la escala satisfacción general con la vida. El procedimiento de respuesta tuvo una duración aproximada de 10 minutos en la muestra inicial y 15 minutos en la muestra adicional. Tanto el instrumento como el procedimiento fueron conocidos y aprobados por el comité de ética científico de la Universidad de Tarapacá.

\section{Análisis Estadístico}

Para establecer evidencia de validez basada en la estructura interna del test se llevó a cabo un modelo de ecuaciones estructurales exploratorias (ESEM), estos modelos permiten estimar el efecto y las relaciones entre variables, dotando a los modelos de regresión de mayor 
flexibilidad al permitir errores de medida tanto en variables dependientes como independientes. Para el ESEM se utilizó rotación GEOMIN (Asparouhouv y Muthén, 2009) y el método robusto de estimación de mínimos cuadrados ponderados (WLSMV), el cual es robusto con variables discretas no normales (Asparouhov y Muthén, 2007), a partir de la matriz de correlaciones policóricas. Para el ajuste general del modelo se consideraron los puntos de cortes propuestos por Schreiber (2017) (e.g CFl> .95, TLl> .95 y RMSEA <.06). Se estimó la fiabilidad para cada dimensión mediante los coeficientes Alpha de Cronbach y omega jerárquico de McDonald. Para evaluar la estabilidad del instrumento entre diferentes sexos (mujeres y hombres) se realizaron las pruebas de invarianza, estas pruebas comprueban que las puntuaciones de los ítems tengan el mismo significado para ambos grupos, osea que no presenten sesgos, para esto se considerado la disminución del CFI (>.005) y el aumento de RMSEA (>.010), como evidencia de invarianza (Chen, 2007). Finalmente, para establecer evidencia de validez basada en su relación con otras variables, se realizó un ESEM de las relaciones entre las dimensiones de la escala EMRS y SWLS-C, la validez convergente prueba que los constructos previamente relacionados en la literatura de hecho, estén relacionados, para esto se utilizó el método de estimación WLSMV y la matriz de correlaciones policóricas.

Los análisis se realizaron mediante el programa Mplus (8.0) (Muthén y Muthén, 2017) y Jamovi (1.8.1) (The Jamovi Project, 2020).

\section{Resultados}

En la tabla 1 se presentan los ajustes de los modelos de medida de ESEM, en su versión original (34 ítems) y en su versión depurada ( 25 ítems).

Tabla 1: Indicadores de ajuste global para la escala multidimensional

\begin{tabular}{ccccccccccc}
\hline Modelo & $N^{\circ}$ Par & $x 2$ & DF & $p$ & CFI & TLI & RMSEA & \multicolumn{2}{c}{ RMSEA } \\
\hline $\begin{array}{c}\text { ESEM } \\
(34)\end{array}$ & 264 & 863.956 & 401 & .00 & .978 & .970 & .054 & .049 & .058 \\
$\begin{array}{c}\text { ESEM } \\
(25)\end{array}$ & 1192 & 354.573 & 185 & .00 & .990 & .983 & .048 & .040 & .055 \\
\hline
\end{tabular}

Nota: $\mathrm{N}$ par: número de parámetros X2: chi-cuadrado; DF: grados de libertad; p: significancia; CFI: índice de ajuste comparativo; TLI: índice de Tucker Lewis; RMSEA: error de aproximación; ESEM 34= Modelo inicial sin depurar; ESEM 25= Modelo final

Si bien el modelo inicial (34 ítems) presenta un ajuste aceptable en los indicadores de medida, según los estándares recomendados en la literatura (e.g. CFl>.95; TLl>.95; RMSEA<.06; Schreiber, 2017). Dado el propósito de generar una escala reducida con una estructura dimensional consistente, buenas representaciones factoriales e ítems unidimensionales, se observaron los índices de modificación y las cargas cruzadas entre algunos ítems de distintas dimensiones (e.i., apoyo social percibido; expectativas al futuro 
y autoestima), optándose por depurar la escala de forma iterativa, basándonos en tres criterios (selección de cargas factoriales fuertes $(\lambda>, 5)$; eliminación de ítems redundantes; y eliminación de reactivos cuyas saturaciones cruzadas fueron mayores a ,3). En base a estos criterios se generó el modelo de 25 ítems.

La escala depurada (25 ítems), se probó mediante ESEM, observándose un buen ajuste (e.g. CFI>.95; TLI>.95; RMSEA<.06; Schreiber, 2017), y la no presencia de cargas factoriales relevantes como se puede ver en la figura 1.

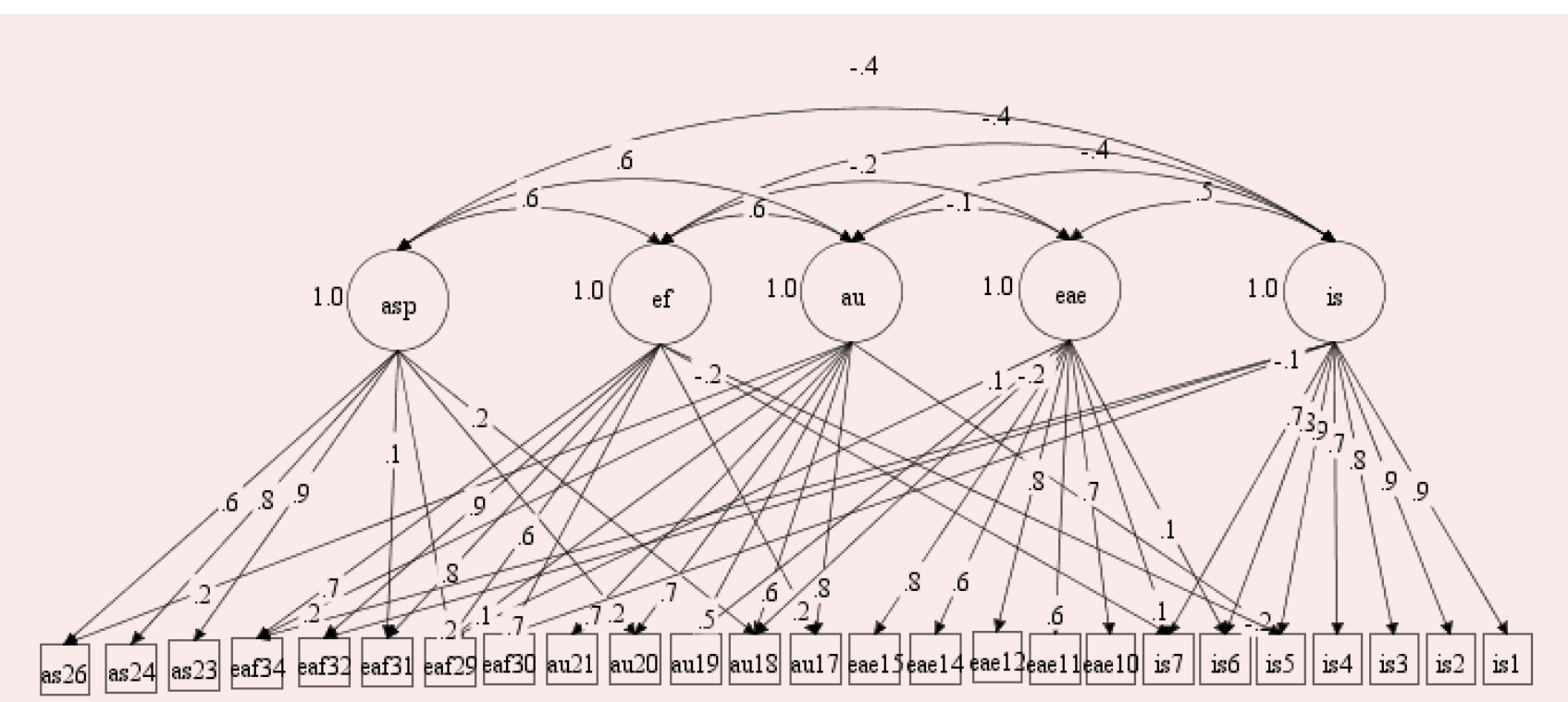

Figura 1: Representación gráfica del modelo ESEM final de 25 ítems y 5 dimensiones.

Nota: La figura muestra las relaciones entre las dimensiones, sus ítems y las respectivas cargas factoriales de cada relación (ESEM). IS = ideación suicida; $E A E$ = Estrategias de afrontamiento enfocadas en la emoción; $E F=$ expectativas hacia el futuro; $A U$ = Autoestima; $A S P=$ Apoyo social percibido;

La escala depurada la componen 25 ítems, diferenciados en 5 dimensiones; Ideación suicida (7 ítems), Estrategias de afrontamiento (5 ítems), Autoestima (5 ítems), Apoyo social percibido (3 ítems), Expectativas hacia el futuro (5 ítems), cuyas saturaciones factorial y estimaciones de fiabilidad (Alpha de Cronbach y coeficiente Omega) se presentan en la tabla 2. 
Desarrollo y evidencia de validez de una escala multidimensional de riesgo de suicidio y factores protectores en un contexto chileno.

Tabla 2: Saturaciones factoriales de la Escala Multidimensional: Ideación Suicida y Factores Protectores Asociados.

\begin{tabular}{|c|c|c|c|c|c|}
\hline & IS & EAE & $\mathrm{EF}$ & $\mathrm{AU}$ & ASP \\
\hline \multicolumn{6}{|l|}{ Ideación Suicida } \\
\hline 1.Pienso en el suicidio como una opción & .939 & -.057 & -.023 & .015 & -.034 \\
\hline 2.Fantaseo con la idea de no estar vivo & .921 & .012 & .007 & .032 & -.001 \\
\hline 3.Estar vivo no vale la pena & .776 & .014 & -.075 & -.029 & -.024 \\
\hline 4.Bromeo acerca de quitarme la vida & .657 & .112 & .039 & -.054 & .040 \\
\hline 5.Atentaría contra mi vida & .861 & -.048 & .133 & -.187 & -.054 \\
\hline 6.Pienso en desaparecer & .808 & .121 & -.049 & -.002 & .023 \\
\hline 7.Me siento aburrido de la vida & .704 & .145 & -.174 & .026 & .007 \\
\hline \multicolumn{6}{|c|}{ Estrategias de Afrontamiento enfocadas en la emoción } \\
\hline 10.El enojo me supera & -.018 & .732 & -.057 & .042 & -.120 \\
\hline 11.La felicidad me descontrola & -.008 & .588 & .113 & -.160 & .090 \\
\hline 12.Las emociones me sobrepasan & .067 & .807 & -.013 & -.027 & -.007 \\
\hline 14. Hay emociones que no se describir & -.002 & .553 & .059 & .043 & -.045 \\
\hline 15.Mis emociones me dominan & .029 & .827 & -.023 & -.031 & .009 \\
\hline \multicolumn{6}{|l|}{ Expectativas hacia el futuro } \\
\hline 29. Tengo objetivos/metas a largo plazo & -.018 & .121 & .614 & -.032 & .165 \\
\hline $\begin{array}{l}\text { 30.Cuando pienso en el futuro me siento } \\
\text { tranquilo }\end{array}$ & -.108 & -.058 & .744 & -.015 & .018 \\
\hline 31.El futuro me parece interesante & .059 & -.071 & .809 & .063 & .138 \\
\hline 32.Percibo el futuro con optimismo & -.124 & -.021 & .856 & .060 & .010 \\
\hline $\begin{array}{l}\text { 34.Sé que puedo alcanzar mis objetivos } \\
\text { próximos }\end{array}$ & -.066 & .053 & .717 & .238 & -.050 \\
\hline \multicolumn{6}{|l|}{ Autoestima } \\
\hline 17. Tengo cualidades positivas & .009 & .016 & .181 & .802 & -.027 \\
\hline 18. Me agrada mi forma de ser & -.057 & -.096 & .046 & .617 & .221 \\
\hline $\begin{array}{l}19 \text { Me siento a gusto con mi apariencia } \\
\text { física }\end{array}$ & -.103 & -.156 & .063 & .529 & .006 \\
\hline $\begin{array}{l}\text { 20.Considero mis sentimientos } \\
\text { importantes }\end{array}$ & .022 & -.035 & -.044 & .721 & .172 \\
\hline $\begin{array}{l}\text { 21.Merezco el aprecio de los demás } \\
\text { Apoyo social percibido }\end{array}$ & .015 & -.027 & .016 & .665 & .128 \\
\hline $\begin{array}{l}\text { 23.Si necesito ayuda o apoyo sé que } \\
\text { puedo contar con un amigo }\end{array}$ & .012 & -.032 & -.043 & .000 & .894 \\
\hline $\begin{array}{l}\text { 24.Me siento parte de una comunidad o } \\
\text { grupo }\end{array}$ & -.050 & -.010 & .045 & .040 & .760 \\
\hline $\begin{array}{l}\text { 26.Sé que cuento con personas que } \\
\text { quieren escucharme }\end{array}$ & -.047 & .041 & .095 & .250 & .638 \\
\hline \multicolumn{6}{|l|}{ Covariaciones Factoriales } \\
\hline $\begin{array}{l}\text { Ideación Suicida } \\
\text { Estrategias de Afrontamiento }\end{array}$ & $.501^{* *}$ & - & & & \\
\hline Expectativas hacia el futuro & $-.415^{* *}$ & $-.162^{* *}$ & - & & \\
\hline Autoestima & $-444^{* *}$ & $-.143^{* *}$ & $.622^{* *}$ & - & \\
\hline Apoyo Social Percibido & $-.381^{* *}$ & -.086 & $.577^{* *}$ & $.614^{* *}$ & - \\
\hline Alpha de Cronbach & .915 & .793 & .802 & .757 & .890 \\
\hline Coeficiente Omega & .920 & .802 & .808 & .764 & .893 \\
\hline
\end{tabular}

$* p \leqslant, 05$

${ }^{* *} \mathrm{p} \leqslant, 01$

Nota: IS = ideación suicida; EAE = Estrategias de afrontamiento enfocadas en la emocion; EHF = Expectativas hacia el futuro; $A U$ = Autoestima; $A S P=$ Apoyo social percibido; se han resaltado las cargas factoriales mayores a 0.3 . 
Las saturaciones factoriales indican que los ítems son representaciones adecuada $(\lambda>0,5)$ de sus dimensiones y presentan bajos niveles de saturaciones cruzadas $(\lambda \leqslant 0,3)$. Para cada dimensión del instrumento, las estimaciones de fiabilidad son óptimas $(\omega>0,8$; Cho y Kim, 2014) o, al menos, suficientes ( $a>0,70$; Cho y Kim, 2014).

Tabla 3: Contraste de Invarianza de medida

\begin{tabular}{llcccccccccc}
\hline & $\mathrm{N}$ par & $\chi^{2}$ & $\mathrm{DF}$ & $\mathrm{P}$ & $\mathrm{CFI}$ & $\mathrm{RMSEA}$ & $\Delta_{\chi^{2}}$ & $\Delta_{\mathrm{DF}}$ & $\mathrm{P}_{\Delta X^{2}}$ & $\Delta_{\text {CFI }}$ & $\Delta_{\mathrm{RMSEA}}$ \\
\hline Configural & 220 & 887.185 & 530 & .000 & .978 & .058 & & & & & \\
Métrico & 200 & 909.029 & 550 & .000 & .978 & .057 & 98.987 & 65 & .001 & .000 & .001 \\
Escalar & 155 & 959.973 & 595 & .000 & .977 & .055 & 71.336 & 45 & .138 & .001 & .003 \\
\hline
\end{tabular}

Nota: $\mathrm{N}$ par: número de parámetros X2: chi-cuadrado; DF: grados de libertad; $\mathrm{p}$ : significación; $\Delta \mathrm{X} 2$ : cambio en chi-cuadrado; $\Delta \mathrm{gl}$ : cambio en los grados de libertad; $\Delta \mathrm{p}$ : cambio de significación.

La tabla 3, muestra los resultados de la prueba de invarianza, en la versión depurada del instrumento ( 25 ítems), para este análisis se utilizó como variable de agrupación el sexo de los participantes (Hombre y Mujer), excluyéndose del análisis los participantes de otros géneros, por razones de potencia estadística. Se observa, que en los diferenciales de ajuste ( $\triangle C F I$ y $\triangle \mathrm{RMSEA}$ ) respecto al modelo de configuración, no hay cambios relevantes, tanto para el modelo métrico $(\triangle \mathrm{CFI}=0,001 ; \triangle \mathrm{RMSEA}=0,001)$ como escalar $(\Delta \mathrm{CFI}=0,000 ; \triangle \mathrm{RMSEA}=0,002)$, dando evidencia de invarianza, dado que no hay una reducción en el diferencial de CFI 0,01, ni un aumento relevante en RMSEA (Chen, 2007). Se establece, que los factores evaluados significan lo mismo tanto para hombres como mujeres, por tanto, se pueden hacer comparaciones entre las puntuaciones de los grupos observados.

Tabla 4: Efectos conjuntos de las dimensiones latentes de la Escala multidimensional de riesgo de suicidio con la Escala de Satisfacción Vital, como variable latente.

\begin{tabular}{llllll}
\hline & IS & EAE & AU & ASP & EHF \\
\hline SWL & $-.379^{*}$ & $-.192^{*}$ & .057 & $.315^{*}$ & $.379^{*}$ \\
\hline
\end{tabular}

Nota: Valores significativos se marcan en negrita ( $p>05)$; IS = ideación suicida; EAE = Estrategias de afrontamiento enfocadas en la emoción ; EHF = expectativas hacia el futuro; $A U$ = Autoestima; $A S P=A p o y o$ social percibido; SWL= The satisfaction with the life scale

Finalmente, la tabla 4 presenta las relaciones entre EMRS y SWL. El modelo presentó un buen ajuste $(C F I=0,974, T L I=0,971$ Y RMSEA =0,051). Se observa un efecto inverso leve $(r<-0,38)$ entre la ideación suicida y SWL. Un efecto inverso bajo entre Estrategias de afrontamiento enfocadas a la emoción y SWL $(r<-0,19)$; También se reportan efectos directos leves entre la SWL y el apoyo social percibido $(r<0,31)$; Así como, en la relación entre SWL y expectativas hacia el futuro donde se observó un efecto directo y leve $(r<0,38)$. Finalmente, la relación entre autoestima y SWL no reportó efectos estadísticamente significativos. 
Desarrollo y evidencia de validez de una escala multidimensional de riesgo de suicidio y factores protectores en un contexto chileno.

\section{Discusión}

El presente estudio se centró en la elaboración de un instrumento psicométrico multidimensional, para evaluar factores vinculados con intentos de suicidio. Los resultados obtenidos en el modelo global, mediante ESEM, la magnitud de las saturaciones factoriales y la inexistencia de saturaciones estadísticamente significativas, permiten sostener la estructura propuesta como explicaciones de las covariaciones entre las respuestas observadas. Así mismo, los coeficientes de fiabilidad obtenidos, para cada dimensión, son óptimos $(\omega>8) \circ$, al menos, suficientes $(a>0,7)$, indicando que la presente escala cuenta con adecuados niveles de consistencia interna (Cho y Kim, 2014). Respecto a los análisis de invarianza, los resultados dan cuenta que es posible utilizar la escala tanto en hombres como en mujeres, en la medida que se pueden hacer comparaciones entre estos grupos a partir de las puntuaciones obtenidas (Chen, 2007). No obstante, es necesario señalar que la invarianza no hace referencia a diferencias de medias, siendo posible, e incluso esperable, que hombres y mujeres presenten diferencias medias en los niveles de rasgo evaluados. En cuanto a la evidencia de validez basada en la relación con otras variables, se observaron relaciones estadísticamente distintas de cero entre satisfacción con la vida con apoyo social percibido, expectativas hacia el futuro, ideación suicida y estrategias de afrontamiento emocionales, lo cual es consistente con la literatura.

En un estudio realizado por Chavarría y Barra (2014), en adolescentes chilenos, se encontró que el apoyo social percibido estaría relacionado con la satisfacción vital, ya que quienes se perciben apoyados presentan mayor satisfacción con la vida (García-Cid et al., 2018; Plexico et al., 2019). En este mismo sentido, las expectativas hacia el futuro se han relacionado con satisfacción con la vida, ya que los planes y aspiraciones son de relevancia en periodos de transición y preparación para el futuro, produciendo sentimientos de control que impactan en la satisfacción vital de las personas, así como también en áreas específicas (e.g. a nivel personal, académico y social) (Azizli et al., 2015). Por otra parte, una baja satisfacción con la vida ha evidenciado ser un predictor de mayor ideación suicida, ya que se suele comprender a la satisfacción con la vida como un componente cognitivo-emocional del bienestar subjetivo (Amer y Hamdan-Mansour, 2014; Yao et al., 2014; Morales-Vives y Dueñas, 2018). En este mismo sentido, se observó una relación inversa estadísticamente significativa, entre las estrategias de afrontamiento emocionales y la satisfacción con la vida, estos resultados concuerdan con lo encontrado en la literatura, cuando se presentan este tipo de estrategias (Guerreiro et al., 2013). Finalmente, cabe señalar que la autoestima no presentó una relación significativa con la satisfacción a la vida, esto puede deberse a la naturaleza mediadora que tiene el autoestima sobre otros factores que inciden en la satisfacción con la vida (e.g. inteligencia emocional, apoyo social percibido) (Chen et al., 2017; Yildiz y Karadas, 2017; Ruvalcaba-Romero et al., 2017), por tanto el efecto del autoestima puede variar de modo condicional ante los niveles observados con otras variables, lo que puede encubrir sus potenciales efectos. 
Entre las principales limitaciones del estudio se encuentra el contexto de pandemia que transcurrió durante la aplicación del cuestionario al segundo grupo encuestado, la que pudo haber impactado en variables demográficas y psicológicas (Urzúa et al., 2020). También es relevante considerar, la crisis social que se vivió en Chile durante toda la aplicación de la encuesta y creación del estudio. De igual manera, el estudio cuenta con una población no representativa del contexto nacional, ya que más de la mitad de los encuestados $(51,4 \%)$, pertenecen a la ciudad de Arica, futuras investigaciones podrían aplicar el instrumento en diversos contextos con la finalidad de aumentar su generalidad y obtener evidencia adicional de validez y representatividad.

Pese a las limitaciones, el presente estudio proporciona un instrumento breve con evidencias psicométricas robustas y actualizadas para su uso en jóvenes y adultos jóvenes con características equivalentes a la muestra. El cual puede ser especialmente útil en contextos de salud y ser un apoyo en los programas de prevención.

\section{Conclusiones}

La importancia de esta investigación radica en la construcción de una escala breve que considera factores protectores (i.e. autoestima, apoyo social percibido, expectativas hacia el futuro) y factores de riesgo (i.e. ideación suicida, estrategias de afrontamiento enfocadas en la emoción) asociados al riesgo suicida, otorgando una visión más amplia e integrativa del fenómeno, lo cual contribuye a generar nuevas estrategias de prevención y detección temprana. El instrumento desarrollado muestra ser útil tanto en su aplicación, como en otorgar conocimiento y un marco referencial para generar nuevas investigaciones, en torno a otras poblaciones.

\section{Referencias}

Akhtar Qureshi, N., Abdullah Alduraihem, R., Mohammad Aldosari, S. y Abdullah Alhabeeb, A. (2020). Tendencia epidemiológica, factores de riesgo, estrategias de prevención y tratamiento de la conducta violenta autodirigida: una evaluación crítica de la literatura relevante. Revista de avances en medicina e investigación médica, 32(1), 51-70. https://doi.org/10.9734/jammr/2020/v32i130350

Alarcón-Vásquez, Y., Bahamón, M. J., Trejos Herrera, A. M., González-Gutiérrez, O., Rubio-

Castro, R., Hernández-Cervantes, Q., \& Gómez-Maqueo, E. L. (2019). Análisis de las propiedades psicométricas del inventario de riesgo suicida (IRISA) para adolescentes colombianos. https://doi.org/10.21865/RIDEP51.2.07

Álvarez, Carola, A., Briceño, Ana, M., Álvarez, K., Abufhele, M., y Delgado, I. (2018). Estudio de adaptación y validación transcultural de una escala de satisfacción con la vida para adolescentes. Revista chilena de pediatría, 89(1), 51-58. https://dx.doi.org/10.4067/ S0370-41062018000100051 
Ambrus, L., Sunnqvist, C., Asp, M., Westling, S. y Westrin, Å. (2020). Coping and suicide risk in high risk psychiatric patients. Journal of Mental Health, 29(1) 27-32. https://doi.org/10.1080/09638237.2017.1417547

Amer N. R. Y., \& Hamdan-Mansour A. M. (2014). Psychosocial predictors of suicidal ideation in patients diagnosed with chronic illnesses in Jordan. Issues in Mental Health Nursing, 35(11), 864-871. https://doi.org/10.3109/01612840.2014.917752

American Educational Research Association, American Psychological Association, y National Council on Measurement in Education. (2014). Standards for educational and psychological testing. American Educational Research Association.

Andrade Salazar, J. A. (2012). Aspectos psicosociales del comportamiento suicida en adolescentes. Revista electrónica de psicología iztacala, 15(2), 688-719. https://tinyurl.com/ $34 \mathrm{sm} 4 \mathrm{tvw}$

Arnett, J. J. (2018). Adolescence and emerging adulthood: A cultural approach. Pearson.

Asparouhouv, T., \& Muthén, B. O. (2007). Wald test of mean equality for potential latent class predictors in mixture modeling. Technical Appendix, 1-3. https://tinyurl.com/2dmyb2du

Asparouhov, T., \& Muthén, B. (2009). Exploratory structural equation modeling. Structural Equation Modeling: A Multidisciplinary Journal, 16(3), 397-438. https://doi.org/10.1080/ 10705510903008204

Austenfeld, J. L., \& Stanton, A. L. (2004). Coping through emotional approach: a new look at emotion, coping, and health-related outcomes. Journal of personality, 72(6), 1335-1364. https://doi.org/10.1111/j.1467-6494.2004.00299.x

Azizli, N., Atkinson, B., E., Baughman, H., M. y Giammarco, E., A. (2015). Relaciones entre autoeficacia general, planificación para el futuro y satisfacción con la vida. Personalidad y diferencias individuales, 82, 58-60. https://doi.org/10.1016/j.paid.2015.03.006

Bachmann, S. (2018). Epidemiología del suicidio y perspectiva psiquiátrica. Revista Internacional de investigación ambiental y salud pública, 15. https://doi.org/gfb424

Bandura, A. (2006). Toward a psychology of human agency. Perspectives on psychological science, 1(2), 164-180. https://doi.org/10.1111/j.1745-6916.2006.00011.x

Barrera, M. (1986). Distinctions between social support concepts, measures, and models. American journal of community psychology, 14(4), 413-445 (1986). https://doi.org/10.1007/ BF00922627 
Beck, A. T., Steer, R. A., y Ranieri, W. F. (1988). Scale for suicide ideation: Psychometric properties of a self-report version. Journal of clinical psychology, 44(4), 499-505. https://doi.org/bjnm9n

Bonilla-Cruz, N. J., Forgiony-Santos, J., Hernández-Lalinde, J., Carrillo-Sierra, S. M., Rivera-Porras, D., Nuván-Hurtado, I. L., y Martínez-Santana, M. C. (2018). Apoyo social percibido en el abordaje terapéutico de adolescentes con orientación suicida. Archivos venezolanos de farmacología y terapéutica, 37(5), 518-522 https://tinyurl.com/ 24zmhubf

Botella, C., Molinari, G., Fernández-Álvarez, J., Guillen, V., García-Palacios, A., Baños, R. M., y Tomás, J. M. (2018). Development and validation of the openness to the future scale: a prospective protective factor. Health and quality of life outcomes, 16(1), 1-16. https://doi.org/10.1186/s12955-018-0889-8

Brausch, A. M., Decker, K. M. (2014). Self-esteem and social support as moderators of depression, body image, and disordered eating for suicidal ideation in adolescents. Journal of abnormal child psychology, 42, 779-789 (2014). https://doi.org/10.1007/s10802-0139822-0

Cabras, C. y Mondo, M. (2017). Coping strategies, optimism, and life satisfaction among firstyear university students in Italy: gender and age differences. Higher education, 75, 643654 (2018). https://doi.org/10.1007/s10734-017-0161-x

Cáceda, R. (2014). Comportamiento suicida: factores de riesgo y de protección. Revista de Neuro-Psiquiatría, 77(1), 3-18. https://doi.org/10.20453/rnp.v77i1.1159

Cai, Z., Chang, Q. y Yip, P.S. (2020). Un análisis cienciométrico de la investigación sobre el el suicidio: 1990-2018. Revista de trastornos afectivos, 266, 356-365. https://doi.org/ 10.1016/j.jad.2020.01.121

Calderón-de la Cruz, G., Merino-Soto, C. y Medina-Zuñiga, P. R. (2019). Validez estructural de la Escala de Apoyo Social en el Trabajo en peruanos. Ansiedad y estrés, 25(2), 148 154. https://doi.org/10.1016/j.anyes.2019.08.001

Cañón, S. C., y Carmona, J. A. (2018). Ideación y conductas suicidas en adolescentes y jóvenes. Pediatría atención primaria, 20(80), 387-397. https://tinyurl.com/vtvs5ecb

Carver, C. S. (1997). You want to measure coping but your protocol' too long: Consider the brief cope. International journal of behavioral medicine, 4(1), 92-100. https://doi.org/ 10.1207/s15327558ijbm0401_6 
Chan, M.K., Bhatti, H., Meader, N., Stockton, S., Evans, J., O'Connor, R.C., Kapur, N., y Kendall, T T. (2016). Predicting suicide following self-harm: Systematic review of risk factors and risk scales. British journal of psychiatry, 209(4), 277-283. https://doi.org/gdegkw

Chang, E. C., Martos, T., Sallay, V., Chang, O. D., Wright, K. M., Najarian, A. S.-M., \& Lee, J. (2016). Examining optimism and hope as protective factors of suicide risk in Hungarian College students: Is risk highest among those lacking positive psychological protection? Cognitive therapy and research, 41(2), 278-288. https://doi.org/gfvrf9

Chavarría, M. P., y Barra, E. (2014). Satisfacción Vital en Adolescentes: Relación con la autoeficacia y el apoyo social percibido. Terapia psicológica, 32(1), 41-46. https://doi.org/10.4067/s0718-48082014000100004

Cheavens, J. S., Cukrowicz, K. C., Hansen, R., y Mitchell, S. M. (2016). Incorporating resilience factors into the interpersonal theory of suicide: The role of hope and self-forgiveness. Journal of clinical psychology, 72, 58-69. https://doi.org/10.1002/jclp.22230

Chen, F. F. (2007). Sensitivity of goodness of fit indexes to lack of measurement invariance. Structural equation modeling: A multidisciplinary journal, 14(3), 464-504. http://dx.doi.org/10.1080/10705510701301834

Chile, Ministerio de Salud (2011). Estrategia Nacional de Salud para el cumplimiento de los objetivos sanitarios de la década 2011-2020. https://tinyurl.com/mkn8wutn

Chile, Ministerio de Salud (2013). Programa Nacional de Prevención del Suicidio: Orientaciones para su implementación. https://tinyurl.com/pkfhtvp9

Cho, E., y Kim, S. (2014). Cronbach's coefficient alpha: Well-known but poorly understood. Organizational research methods, 18(2), 207-230. https://doi.org/gft9fz

Claes, L., Luyckx, K., Baetens, I., Van de Ven, M. y Witteman, C. (2015). Bullying and victimization, depressive mood, and non- suicidal self-injury in adolescents: the moderating role of parental support. Journal of child and family studies, 24(11), 3363-3371. https://doi.org/10.1007/s10826-015-0138-2

Cohen, S., y Wills, T. A. (1985). Stress, social support, and the buffering hypothesis. Psychological bulletin, 98(2), 310-357. https://doi.org/10.1037/0033-2909.98.2.310

Cooley, J. L., Fite, P. J., Rubens, S. L. y Tunno, A. M. (2015). Peer victimization, depressive symptoms, and rule-breaking behavior in adolescence: the moderating role of peer social support. Journal of psychopathology and behavioral assessment, 37(3), 512-522. https://doi.org/10.1007/s10862-014-9473-7 
Crosby, A. E., Ortega, L., Melanson, C. (2011). Vigilancia de la violencia autodirigida: definicioes uniformes y elementos de datos recomendados, versión 1.0. Atlanta (GA): Centros para el Control y Prevención de Enfermedades, Centro Nacional para la Prevención y Control de Lesiones; 2011. https://tinyurl.com/7wefzd

Davidson, L. M. y Demaray, M. K. (2007). Social support as a moderator between victimization and internalizing-externalizing distress from bullying. School psychology review, 36(3), 383-405. https://doi.org/10.1080/02796015.2007.12087930

Demirtepe-Saygli, D. y Bozo, Ö. (2011). Perceived social support as a moderator of the relationship between caregiver well- being indicators and psychological symptoms. Journal of health psychology, 16(7), 1091-1100. https://doi.org/10.1177/1359105311399486

Denis-Rodríguez, E., Barradas Alarcón, M. E., Delgadillo-Castillo, R., Denis-Rodríguez, P. B., y Melo-Santiesteban, G. (2017). Prevalencia de la ideación suicida en estudiantes de Medicina en Latinoamérica: un meta análisis. RIDE. Revista iberoamericana para la investigación y el desarrollo educativo, 8(15), 387-418. https://dx.doi.org/10.23913/ ride.v8i15.304

Deuter, K., Procter, N., y Evans, D. (2019). Protective factors for older suicide attempters: Finding reasons and experiences to live. Death studies. https://doi.org/10.1080/ 07481187.2019 .1578303

Diener, E., Emmons, R. A., Larsen, R. J., y Griffin, S. (1985). The satisfaction with life scale. Journal of personality assessment, 49(1), 71-75. https://doi.org/10.1207/ s15327752jpa4901_13

Durkheim E. (1897). Le suicide: Etude de sociologie. Paris.

Echávarri, O., Morales, S., Barros, J., Armijo, I., Maino, M. P., Núñez, C., Fischman, R., Moya, C., Szmulewicz, M., y Tomicic, A. (2018). Validación de la escala razones para vivir en consultantes a salud mental de la Región Metropolitana de Chile. Psykhe, 27(2). https://doi.org/10.7764/psykhe.27.2.1153

Echávarri, O., Morales, S., Bedregal, P., Barros, J., de la Paz Maino, M., Fischman, R., Peñaloza, F., y Moya, C. (2015). ¿Por qué no me suicidaría? Comparación entre pacientes hospitalizados en un servicio de psiquiatría con distinta conducta suicida. Psykhe, 24(1), 1-11. https://doi.org/10.7764/psykhe.24.1.667

Echávarri, O, Maino, M. P., Fischman, R, Morales, S., y Barros, J. (2015b). Aumento sostenido del del suicidio en Chile: Un tema pendiente. Temas de la agenda pública, 10(79), 3-14. https://tinyurl.com/dj2f4uwd 
Encuesta Nacional de Salud (2017). Segunda entrega de resultados. Chile. https://tinyurl.com /6fr5jzph

Eryılmaz, A. (2011). The relationship between adolescents' subjective well-being and positive expectations towards future. Dusunen Adam the journal of psychiatry and neurological sciences, 24(3), 209-215. https://doi.org/10.5350/DAJPN2011240306

Evans, S. E., Steel, A. L. y DiLillo, D. (2013). Child maltreatment severity and adult trauma symptoms: Does perceived social support play a buffering role?. Child abuse \& neglect, 37(11), 934-943. https://doi.org/10.1016/j.chiabu.2013.03.005

Fernández-Abascal, E. G. y Palmero F. (1999). Emociones y salud. Ariel.

Fleming, J. S., y Courtney, B. E. (1984). The dimensionality of self-esteem: II. Hierarchical facet model for revised measurement scales. Journal of personality and social psychology, 46(2), 404. https://doi.org/10.1037/0022-3514.46.2.404

Folkman, S., y Lazarus, R. S. (1984). Stress, appraisal, and coping. Springer.

Folkman, S., y Lazarus, R. S. (1988). Coping as a mediator of emotion. Journal of personality and social psychology, 54(3), 466. https://doi.org/10.1037/0022-3514.54.3.466

García-Cid, A., Hombrados-Mendieta, I., Gómez-Jacinto, L., Palma-García, M. D. las O., y Millán-Franco, M. (2018). Apoyo social, resiliencia y región de origen en la salud mental y la satisfacción vital de los inmigrantes. Universitas psychologica, 16(5), 1-14. https://doi.org/10.11144/Javeriana.upsyl6-5.asrr

Guerreiro, D. F., Cruz, D., Frasquilho, D., Santos, J. C., Figueira, M. L., y Sampaio, D. (2013). Association between deliberate self-harm and coping in adolescents: a critical review of the last 10 years' literature. Archives of suicide research, 17(2), 91-105. https://doi.org/10.1080/13811118.2013.776439

Heberle, A.E., Krill, S.C., Briggs-Gowan, M.J. y Carter, A. S. (2015). Predicting externalizing and internalizing behavior in kindergarten: examining the buffering role of early social support. Journal of clinical child \& adolescent psychology, 44(4), 640-654. https://doi.org/10.1080/15374416.2014.886254

Hirsch, J. K., y Kelliher Rabon, J. (2015). Optimistic explanatory style and suicide attempt in young adults. International journal of mental health and addiction, 13, 675-686. https://doi.org/10.1007/s11469-015-9570-1 
Hirsch, J. K., y Kelliher Rabon, J. (2015). Optimistic explanatory style and suicide attempt in young adults. International Journal of mental health and addiction, 13, 675-686. https://doi.org/10.1007/s11469-015-9570-1

Huffman, J. C., Boehm, J. K., Beach, S. R., Beale, E. E., DuBois, C. M., y Healy, B. C. (2016). Relationship of optimism and suicidal ideation in three groups of patients at varying levels of suicide risk. Journal of psychiatric research, 77, 7776-7784. https://doi.org/10.1016/ j.jpsychires.2016.02.020

Interian, A., Chesin, M., Kline, A., St. Hill, L., King, A., Miller, R., Latorre, M., Gara, M., y Stanley, B. (2019). Coping with suicidal urges: An important factor for suicide risk assessment and Intervention. Archives of suicide research, 25(2), 224-237. https://doi.org/10.1080/ 13811118.2019.1678538

King, J. D., y Kowalchuk, B. (1994). ISO 30.Adolescent inventory of suicide orientation-30. National Computer Systems.

Krach, S. K., McCreery, M. P., y Guerard, J. (2016). Cultural-linguistic test adaptations: Guidelines for selection, alteration, use, and review. School psychology international, 38(1), 321. https://doi.org/10.1177/0143034316684672

Lazarus, R., y Folkman, S. (1986). Estrés y procesos cognitivos. Martínez Roca.

Liu, R. T., Bettis, A. H., y Burke, T. A. (2020). Characterizing the phenomenology of passive suicidal ideation: a systematic review and meta-analysis of its prevalence, psychiatric comorbidity, correlates, and comparisons with active suicidal ideation. Psychological medicine, 50(3), 367-383. https://doi.org/10.1017/S003329171900391X

Lu, W., y Hampton, K. N. (2016). Beyond the power of networks: Differentiating network structure from social media affordances for perceived social support. New media \& society, 19, 861-879. https://doi.org/10.1177/1461444815621514

Manani, P. y Sharma, S. (2013). Self esteem and suicidal ideation: A correlational study. MIER Journal of educational studies trends \& practices, 3(1), 75-83. https://doi.org/ $10.52634 / \mathrm{mier} / 2013 / \mathrm{v} 3 / \mathrm{il} / 1556$

Martínez, M. J. C., Martínez, M. R., García, J. C., Cortés, M. I. O., Ferrer, A. R., y Herrero, B. T. (2004). Fiabilidad y validez de la Escala de Satisfacción con la Vida de Diener en una muestra de mujeres embarazadas y puérperas. Psicothema, 16(3), 448-455. https://tinyurl.com/cva39833 
Mascayano, F., Irrazabal, M., Emilia, W. D., Vaner, S. J., Sapag, J. C., Alvarado, R., Yang, L. H. y y Sinah, B. (2015). El suicidio en América Latina: un problema creciente de salud pública. Revista de la Facultad de Ciencias Médicas, 72(4) , 295-303. https://dx.doi.org/10.31053/1853.0605.v72.n4.13837

Mokdad, A. H., Forouzanfar, M. H., Daoud, F., Mokdad, A. A., El Bcheraoui, C., Moradi-Lakeh, M., Kyu, H. H., Barber, R. M., Wagner, J., Cercy, K., Kravitz, H., Coggeshall, M., Chew, A., O'Rourke, K. F., Steiner, C., Tuffaha, M., Charara, R., Al-Ghamdi, E. A., Adi, Y., ... Murray, C. J. (2016). Global burden of diseases, injuries, and risk factors for Young People's health during 1990-2013: A systematic analysis for the global burden of disease study 2013. The Lancet, 387(10036), 2383-2401. https://doi.org/10.1016/s01406736(16)00648-6

Morales-Vives, F., y Dueñas, J. M. (2018). Predicting suicidal ideation in adolescent boys and girls: the role of psychological maturity, personality traits, depression and life satisfaction. The Spanish journal of psychology, 21. https://doi.org/10.1017/sjp.2018.12

Muñoz de Aranillas, R., Borrero, M. F., Moreno, P. P., \& Bollido, M. F. (2010). Evidencias de validez de la adaptación al español de la escala de satisfacción con la vida (SWLS) en una muestra de estudiantes universitarios. Metodología de encuestas, 12(1), 45-62. https://tinyurl.com/3uhb75ap

Muthén, L. K. y Muthén, B. (2017, Abril 20). MPlusVersion (8). Mplus Home Page. Muthén y Muthén. https://www.statmodel.com/

O'Connor, M., Dooley, B. y Fitzgerald, A. (2014).constructing the suicide risk index (sri): does it work in predicting suicidal behavior in young adults mediated by proximal factors? Archives of suicide research, 19(1), 1-16. https://doi.org/10.1080/13811118.2014.915775

O'Connor, R. C. y Nock, M. K. (2014). The psychology of suicidal behaviour. The Lancet Psychiatry, 1(1), 73-85. https://doi.org/10.1016/S2215-0366(14)70222-6

Organisation for Economic Co-operation and Development. (2014). Suicides. En OECD Factbook 2014: Economic, Environmental and Social Statistics (pp. 240-241). OECD. https://doi.org/10.1787/factbook-2014-99-en

Organisation for Economic Co-operation and Development. (2019). Society at a glance 2019: OECD Social Indicators. OECD. https://doi.org/10.1787/soc_glance-2019-en

Organisation for Economic Co-operation and Development. (2020). Suicide rates [indicator]. https://doi.org/10.1787/bd12d298-en 
Okasha, A., Lotaif, F. y Sadek, A. (1981). Prevalence of suicidal feelings in a sample of non-consulting medical students. Acta psychiatrica scandinavica, 63(5), 409-415. https://doi.org/10.1111/j.1600-0447.1981.tb00690.x

Organización mundial de la Salud. (2019). Suicidio. https://tinyurl.com/52uxweu3

Ortiz, E, Meza Meza, M. y Cañón, S. (2019) Determinación de los factores de riesgo asociados a la conducta suicida en adolescentes. Scipedia. Collection of Articles on Medicine, (1). https://tinyurl.com/2sxn3ys9

Otzen, T., Fuentes, N., Wetzel, G., Henríquez, C., Antúnez, Z., y Melnik, T. (2020). Suicidality and perceived social support in university students with chronic non-communicable diseases. Terapia psicológica, 38(1), 119-129. https://dx.doi.org/10.4067/S071848082020000100119

Patterson, W. M., Dohn, H. H., Bird, J., y Patterson, G. A. (1983). Evaluation of suicidal patients: the SAD PERSONS scale. Psychosomatics, 24(4), 343-349. https://doi.org/10.1016/ S0033-3182(83)73213-5

Paykel, E., Myers, J., Lindenthal, J., y Tanner, J.(1974).Suicidal feelings in the general population: A prevalence study. British journal of psychiatry, 124(582), 460-469. https://doi.org/10.1192/bjp.124.5.460

Pedrosa, I., García-Cueto, E., Suárez-Álvarez, J., y Sánchez, B. P. (2012). Adaptación española de una Escala de Apoyo Social percibido para deportistas. Psicothema, 24(3), 470476. https://tinyurl.com/53v8csxm

Perczek, R., Carver, C. S., Price, A.A., y Pozo-Kaderman, C. (2000). Coping, mood, and aspects in Spanish translations and evidence of convergence with English versions. Journal of personality assessment, 74, 63-87. https://doi.org/10.1207/S15327752JPA740105

Pierce, D. W. (1977). Suicidal intent in self-injury. The British journal of psychiatry, 130(4), 377385. https://doi.org/10.1192/bjp.130.4.377

Plexico, L. W., Erath, S., Shores, H., y Burrus, E. (2019). Self-acceptance, resilience, coping and satisfaction of life in people who stutter. Journal of fluency disorders, 59, 52-63. https://doi.org/10.1016/j.jfludis.2018.10.004

Raffaelli, M., Andrade, F. C., Wiley, A. R., Sanchez-Armass, O., Edwards, L. L., y Aradillas-García, C. (2013). Stress, social support, and depression: a test of the stress-buffering hypothesis in a Mexican sample. Journal of research on adolescence, 23(2), 283-289. https://doi.org/10.1111/jora.12006 
Ronen, T., Hamama, L., Rosenbaum, M., y Mishely-Yarlap, A. (2014). Subjective well-being in adolescence: The role of self-control, social support, age, gender, and familial crisis. Journal of happiness studies, 17(1), 81-104. https://doi.org/10.1007/s10902-014-9585-5

Rosenberg, M. (1965). Society and the adolescent self-image. Princeton University. https:// tinyurl.com/vmjak669

Rosenstiel, A. K., y Keefe, F. J. (1983). The use of coping strategies in chronic low back pain patients: relationship to patient characteristics and current adjustment. Pain, 17(1), 33-44. https://doi.org/10.1016/0304-3959(83)90125-2

Rossi, J. L., Jiménez, J. P., Barros, P., Assar, R., Jaramillo, K., Herrera, L., Quevedo, Y., Botto, A., Leighton, C., y Martínez, F. (2019). Sintomatología depresiva y bienestar psicológico en estudiantes universitarios chilenos. Revista médica de chile, 147(5), 579-588. https://doi.org/10.4067/s0034-98872019000500579

Ruvalcaba-Romero, N. A., Fernández-Berrocal, P., Salazar-Estrada, J. G., y Gallegos-Guajardo, J. (2017). Positive emotions, self-esteem, interpersonal relationships and social support as mediators between emotional intelligence and life satisfaction. Journal of behavior, health \& social issues, 9(1), 1-6. https://doi.org/10.1016/j.jbhsi.2017.08.001

Sarid, O., Slonim-Nevo, V., Pereg, A., Friger, M., Sergienko, R., Schwartz, D., Greenberg, D., Shahar, I., Chernin, E., Vardi, H., Eidelman, L., Segal, A., Ben-Yakov, G., Gaspar, N., Munteanu, D., Rozental, A., Mushkalo, A., Dizengof, V., Abu-Freha, N., ... Odes, S. (2017). Coping strategies, satisfaction with life, and quality of life in crohn's disease: A gender perspective using structural equation modeling analysis. Plos One, 12(2). https://doi.org/10.1371/journal.pone.0172779

Scheier, M. F., Carver, C. S., y Bridges, M. W. (1994). Distinguishing optimism from neuroticism (and trait anxiety, self-mastery, and self-esteem): a reevaluation of the life orientation test. Journal of personality and social psychology, 67, 1063-1078. https://doi.org/10.1037/0022-3514.67.6.1063

Schreiber, J. (2017). Update to core reporting practices in structural equation modeling. Research in social and administrative pharmacy, 13(3), 634-643. https://doi.org/gd3vm6

Schütz, A., Rentzsch, K., y Sellin, I. (2006). Multidimensionale selbstwertskala: MSWS; Manual. Hogrefe.

Seginer, R. (2009). Future orientation. Springer. https://doi.org/10.1007/b106810

Shavelson, R.J., Hubner, J.J. y Stanton, G.C. (1976). Self-concept: validation of construct interiretations. Review of educational research, 46(3), 407-441. https://doi.org/bzzjxv 
Simon, G. E., Specht, C., y Doederlein, A. (2016). Coping with suicidal thoughts: a survey of personal experience. Psychiatric services, 67(9), 1026-1029. https://doi.org/f9c268

Siu, A. M. (2019). Self-harm and suicide Among children and adolescents in Hong Kong: a review of prevalence, risk factors and prevention strategies. Journal of adolescent health, 64(6), S59-S64. https://doi.org/10.1016/j.jadohealth.2018.10.004

Smith, E.R., Mackie, D.M., y Claypool, H.M.(2015). Social psychology (4a ed.). Psychology Press.

Solano, P., Aguglia, A., Caprino, M., Conigliaro, C., Giacomini, G., Serafini, G., y Amore, M. (2019). The personal experience of severe suicidal behaviour leads to negative attitudes towards self- and other's suicidal thoughts and behaviours: A study of temperaments, coping strategies, and attitudes towards suicide among medical students. Psychiatry research, 272, 669-675. https://doi.org/10.1016/j.psychres.2018.12.116

Soto-Sanz,V., Piqueras, J. A., Rodríguez-Marín; J., Pérez-Vázquez, M. T., Rodríguez-Jiménez, T., Castellví, P., Miranda-Mendizábal, A., Parés-Badell, O., Almenara, J., Blasco, M. J., y Cebrià, A., (2019). Self-esteem and suicidal behaviour in youth: a meta-analysis of longitudinal studies. Psicothema, 3/(3), 246-254. https://doi.org/gh65p4

Stanley, B., Green, K. L., Ghahramanlou-Holloway, M., Brenner, L. A., \& Brown, G. K. (2017). The construct and measurement of suicide-related coping. Psychiatry research, 258, 189193. https://doi.org/10.1016/j.psychres.2017.08.008.

Steeg, S., Quinlivan, L., Nowland, R., Carroll, R., Casey, D., Clements, C., Cooper, J., Davies, L., Knipe, D., Ness, J., O'Connor, R. C., Hawton, K., Gunnell, D., y Kapur, N. (2018). Accuracy of risk scales for predicting repeat self-harm and suicide: A multicentre, populationlevel cohort study using routine clinical data. BMC Psychiatry, 18(1). https://doi.org/10.1186/s12888-018-1693-z

Surgenor, P. W. G. (2015), Promoviendo la recuperación de la ideación suicida a través del desarrollo de factores protectores. Counselling \& psychotheapy research, 15(1), 207216. https://doi.org/10.1002/capr.12035

Tanigawa, D., Furlong, M. J., Felix, E. D. y Sharkey, J. D. (2011). The protective role of perceived social support against the manifestation of depressive symptoms in peer victims. Journal of school violence, 10(4), 393-412. https://doi.org/fkscs7

The Jamovi project. (2021, Abril, 19). Jamovi (Version 1.8.1). Jamovi. Stats. Open. Now. https://www.jamovi.org

Turecki, G., Brent, D. A., Gunnell, D., O'Connor, R. C., Oquendo, M. A., Pirkis, J., y Stanley, B. H. (2019). Suicide and suicide risk. Nature reviews disease primers, 5(1). https://doi.org/ggjqdb 
Turecki, G., y Brent, D. A. (2016). Suicide and suicidal behaviour. The Lancet, 387(10024), 12271239. https://doi.org/10.1016/S0140-6736(15)00234-2

Urzúa, A., Vera-Villarroel, P., Caqueo-Urízar, A. y Polanco-Carrasco, R.. (2020). Psicología en la prevención y manejo de COVID-19. Contribuciones de la evidencia inicial. Terapia psicológica, 38(1), 103-118. https://dx.doi.org/10.4067/S0718-48082020000100103

Usta, Y. Y. (2012). Importance of social support in cancer patients. Asian pacific journal of of cancer prevention, 13(8), 3569-3572. https://doi.org/10.7314/apjcp.2012.13.8.3569

Yao, Y.-S., Chang, W.-W., Jin, Y.-L., Chen, Y., He, L.-P., y Zhang, L. (2014). Life satisfaction, coping, self-esteem and suicide ideation in Chinese adolescents: A school-based study. Child: care, health and development, 4O(5), 747-752. https://doi.org/10.1111/cch.12142

Yildiz, M. A., y Karadas, C. (2017). Multiple mediation of self-esteem and perceived social support in the relationship between loneliness and life satisfaction. Journal of education and practice, 8(3), 130-139. https://tinyurl.com/4ukxxx78

Yu, E., Cheavens, J., Vilhauer, J., y van Beek, W. (2019). Future-oriented treatments for suicide: an overview of three modern approaches. En J. Hirsch, E. Chang, y J. Kelliher Rabon (Eds.), A positive psychological approach to suicide (Advances in Mental Health and Addiction, pp. 183-208). Springer. https://doi.org/10.1007/978-3-030-03225-8_9

Zhang, J., Liu, Y., y Sun, L. (2017). Psychological strain and suicidal ideation: A comparison between Chinese and US college students. Psychiatry research, 255, 256-262. https://doi.org/10.1016/j.psychres.2017.05.046

\section{Para citar en APA}

Sepúlveda-Páez, G., Escudero-Pastén, J., Santibáñez-Palma, J. F. y Ferrer-Urbina, R. (2021). Desarrollo y evidencia de validez de una escala multidimensional de riesgo de suicidio y factores protectores en un contexto chileno. Terapia Psicológica (En línea), 39(2), 175-198. https://doi.org/10.4067/S0718-48082021000200175 